\title{
Intermediate polars in the Swift/BAT survey: spectra and white dwarf masses
}

\author{
J. Brunschweiger ${ }^{1}$, J. Greiner $^{1}$, M. Ajello ${ }^{1, \star}$, and J. Osborne ${ }^{2}$ \\ 1 Max-Planck-Institut für Extraterrestrische Physik, Giessenbachstrasse 1, 85748 Garching, Germany \\ e-mail: jcg@mpe.mpg.de \\ 2 Dept. of Physics and Astronomy, University of Leicester, University Road, Leicester LE1 7RH, UK \\ e-mail: julo@star.le.ac.uk
}

Received 3 November 2008 / Accepted 27 December 2008

\begin{abstract}
Context. White dwarf masses in cataclysmic variables are difficult to determine accurately, but are fundamental for understanding binary system parameters, as well as binary evolution.

Aims. We investigate the X-ray spectral properties of a sample of Intermediate Polars (IP) detected above $15 \mathrm{keV}$ to derive the masses of their accreting white dwarfs.

Methods. We use data from the Swift/BAT instrument which during the first 2.5 yrs of operation has detected 22 known intermediate polars. The X-ray spectra of these sources are used to estimate the mass of the white dwarfs.

Results. We are able to produce a mass estimate for 22 out of 29 of the confirmed intermediate polars. Comparison with previous mass measurements shows good agreement. For GK Per, we were able to detect spectral changes due to the changes in the accretion rate.

Conclusions. The Swift/BAT detector with its combination of sensitivity and all-sky coverage provides an ideal tool to determine accurate white dwarf masses in intermediate polars. This method should be applied to other magnetic white dwarf binaries.
\end{abstract}

Key words. accretion, accretion disks - stars: binaries: general

\section{Introduction}

Cataclysmic variables $(\mathrm{CV})$ are binaries with a white dwarf (WD) accreting matter from a late main-sequence star which fills its Roche lobe (Warner 1995). Depending on the strength of the magnetic field of the white dwarf, the matter flowing through the Lagrange point either forms a full or partial accretion disk or follows the magnetic field lines towards the magnetic poles of the white dwarf. Intermediate polars (IPs) are a sub-type of cataclysmic variables (see e.g. the review by Hellier 2002) where a magnetic field of order $B \approx 1-10$ MG (de Martino et al. 2004) disrupts the inner part of the accretion disk. From this disruption radius the accreting material follows the magnetic field lines towards the magnetic poles and finally falls freely onto the surface of the WD, forming a strong shock near the surface of the WD (Aizu 1973). This so called post shock region is heated up to a high temperature, about 10-20 keV (King \& Lasota 1979). The main cooling process is thermal Bremsstrahlung. The temperature of the post shock region and hence the spectral shape of the emitted X-ray radiation depend to first order only on the mass of the WD. This makes IPs especially interesting, because their X-ray spectrum allows a relatively simple estimate of the WD mass (see e.g. Rothschild et al. 1981; Ishida 1991; Suleimanov et al. 2005).

The white dwarf mass is a fundamental parameter in cataclysmic binaries. It not only governs the dynamics of the orbital motion and the accretion flow, but also is a main parameter characterizing the emission of the accretion region (see above).

\footnotetext{
* Present address: SLAC/KIPAC, 2575 Sand Hill Road, Menlo Park, CA 94025; majello@slac.stanford.edu
}

Moreover, it plays a fundamental role in the binary evolution, and accurate mass distributions of certain sub-classes can help in constraining the accretion history.

Previous attempts to determine WD masses of IPs using X-ray measurements have been controversial, since data from different X-ray instruments led to different mass estimates (Ishida 1991 with Ginga data; or Ramsay et al. 1998 using RXTE/PCA, ASCA and Ginga data), and in all cases X-ray determined masses where substantially higher than masses derived from optical timing or spectroscopy. The classical example is XY Ari, an eclipsing IP (which is considered to be the most suitable object because the uncertainty in the inclination is very small): the RXTE spectrum yields $1.22 M_{\odot}$, the ASCA data $1.27-1.40 M_{\odot}$ depending on GIS, SIS or a combination of the two detectors, and the Ginga data result in $1.3 M_{\odot}$, while optical methods yield $0.78-1.03 M_{\odot}$ (Ramsay et al. 1998).

Observations above $20 \mathrm{keV}$ are crucial as they provide a clean signal not contaminated by other system components such as the accretion disc or the main-sequence star and it avoids complications due to cold or warm absorbers as well as fluorescence (line) emission. Moreover, at or above this energy, effects of absorption in the post-shock region are negligible. The only effect to take into account is reflection which is known to be present in magnetic cataclysmic variables due to the detection of the $6.4 \mathrm{keV}$ emission line. The only disadvantage was the weakness of the X-ray flux. Thus, a sensitive instrument must be used for this kind of observation. The Swift Burst Alert Telescope (BAT, Barthelmy 2004), with an unprecedented sensitivity in the $15-200 \mathrm{keV}$ range, opened a new window for the study of the hard X-ray sky, combining high sensitivity and all-sky coverage. 
Table 1. IPs detected in the BAT survey $3 \sigma$ in the $15-55 \mathrm{keV}$ band ordered by constellation. The first three columns give the BAT position and the offset to the optical position. The count rate was averaged over the entire $2.5 \mathrm{yr}$ observing period. In the lower part of the table we report those sources which are not currently detected in the BAT survey; for those we give the optical position for which the upper limit was computed.

\begin{tabular}{|c|c|c|c|c|c|c|c|}
\hline Name & $\begin{array}{r}\text { RA } \\
(2000.0)\end{array}$ & $\begin{array}{r}\text { Dec } \\
(2000.0)\end{array}$ & $\begin{array}{r}\text { Offset } \\
\text { (arcmin) }\end{array}$ & $\begin{array}{c}\text { Count rate } \\
\left(10^{-5} \mathrm{cts} / \mathrm{s} / \mathrm{det}\right)\end{array}$ & $\begin{array}{r}\text { Significance } \\
\text { ratio }\end{array}$ & $\begin{array}{l}\text { Exp. time } \\
\text { (Ms) }\end{array}$ & $\begin{array}{c}\text { Sensitivity } \\
\left(10^{-11} \mathrm{erg} / \mathrm{cm}^{2} / \mathrm{s}\right)\end{array}$ \\
\hline FO Aquarii & 334.481 & -8.351 & 3.3 & $12.4 \pm 0.8$ & 16.6 & 2.76 & 1.99 \\
\hline XY Arietis & 44.038 & 19.441 & 4.3 & $5.3 \pm 0.6$ & 7.9 & 2.70 & 1.55 \\
\hline V405 Aurigae & 89.497 & 53.896 & 0.3 & $5.4 \pm 0.6$ & 8.7 & 2.70 & 1.66 \\
\hline MU Camelopardaris & 96.318 & 73.578 & 1.4 & $2.9 \pm 0.5$ & 6.7 & 3.46 & 1.60 \\
\hline V709 Cassiopeiae & 7.204 & 59.289 & 0.7 & $16.2 \pm 0.7$ & 27.5 & 3.65 & 1.61 \\
\hline V1025 Centauri & 189.569 & -38.713 & 4.1 & $1.6 \pm 0.8$ & 3.1 & 1.91 & 1.88 \\
\hline BG Canis Minoris & 112.871 & 9.940 & 2.9 & $5.8 \pm 0.8$ & 7.0 & 1.92 & 2.15 \\
\hline TV Columbae & 82.356 & -32.818 & 2.5 & $13.0 \pm 0.7$ & 20.3 & 3.55 & 1.44 \\
\hline TX Columbae & 85.834 & -41.032 & 3.5 & $4.5 \pm 0.5$ & 7.8 & 3.81 & 1.31 \\
\hline V2306 Cygni & 299.560 & 32.545 & 3.4 & $3.1 \pm 0.5$ & 4.8 & 3.85 & 1.77 \\
\hline DO Draconis & 175.910 & 71.689 & 1.8 & $2.7 \pm 0.4$ & 7.8 & 4.47 & 1.11 \\
\hline PQ Geminorum & 117.822 & 14.740 & 2.7 & $6.2 \pm 0.7$ & 9.4 & 2.04 & 2.17 \\
\hline EX Hydrae & 193.102 & -29.249 & 3.0 & $5.2 \pm 0.7$ & 6.4 & 1.87 & 1.97 \\
\hline NY Lupi & 237.061 & -45.478 & 2.1 & $15.2 \pm 0.8$ & 15.5 & 1.35 & 2.58 \\
\hline V2400 Ophiuchi & 258.152 & -24.246 & 4.2 & $10.5 \pm 0.9$ & 11.0 & 1.30 & 2.52 \\
\hline GK Persei & 52.799 & 43.905 & 4.4 & $18.5 \pm 0.9$ & 30.4 & 2.88 & 1.58 \\
\hline AO Piscium & 343.825 & -3.178 & 4.7 & $8.1 \pm 0.6$ & 11.1 & 3.00 & 1.62 \\
\hline V1223 Sagittarii & 283.759 & -31.163 & 3.0 & $19.6 \pm 1.1$ & 20.8 & 1.31 & 2.54 \\
\hline V1062 Tauri & 75.615 & 24.756 & 5.3 & $3.1 \pm 0.8$ & 5.9 & 2.09 & 2.25 \\
\hline RX J2133.7+5107 & 323.432 & 51.124 & 1.9 & $10.9 \pm 0.6$ & 18.2 & 3.99 & 1.42 \\
\hline Swift J0732.5-1331 & 113.156 & -13.518 & 2.8 & $6.3 \pm 0.6$ & 9.7 & 2.61 & 1.70 \\
\hline IGR J00234+6141 & 5.740 & 61.686 & 5.3 & $2.0 \pm 0.6$ & 3.0 & 3.68 & 1.72 \\
\hline IGR J17303-0601 & 262.590 & -5.993 & 0.8 & $14.0 \pm 0.8$ & 12.7 & 1.59 & 2.78 \\
\hline AE Aquarii & 310.04 & -0.87 & - & $<0.3$ & 0.4 & 2.44 & 2.05 \\
\hline HT Camelopardaris & 119.26 & 63.10 & - & $<0.1$ & 0.3 & 3.52 & 1.34 \\
\hline DW Cancri & 119.72 & 16.28 & - & $<1.5$ & 1.5 & 2.09 & 2.11 \\
\hline UU Columbae & 78.05 & -32.69 & - & $<0.7$ & 1.7 & 3.55 & 1.50 \\
\hline DQ Herculis & 271.88 & -45.86 & - & $<1.0$ & 2.5 & 3.75 & 1.56 \\
\hline WX Pyxidis & 128.27 & -22.81 & - & $<1.4$ & 2.8 & 3.16 & 1.56 \\
\hline
\end{tabular}

${ }^{1}$ Minimum flux to detect a source at $3 \sigma$ at a given position in the $15-55 \mathrm{keV}$ band.

The present paper is organized as follows: in Sect. 2 we introduce the observations. The results of the measurement can be found in Sect. 3 which is divided in three subsections to discuss first the spectral analysis, second the accretion-rate dependence observed in GK Per, and finally the comparison with previous methods of mass determination (both with X-ray and non X-ray methods). The conclusions are given in Sect. 4.

\section{Data and models}

\subsection{The Swift/BAT X-ray survey}

BAT is a coded mask telescope with a wide field of view aperture (FOV, $120^{\circ} \times 90^{\circ}$ partially coded) sensitive in the energy range of 15-195 keV. BAT continuously monitors the hard X-ray sky surveying gamma-ray bursts. Results of the BAT survey (Markwardt et al. 2005; Ajello et al. 2008a) show that BAT reaches a sensitivity of $\approx 1 \mathrm{mCrab}$ in a $1 \mathrm{Ms}$ exposure. With its sensitivity and the large amount of exposure already accumulated over the whole sky, BAT is a very good instrument for studying IPs above $20 \mathrm{keV}$.

In the analysis presented here we use BAT data to derive spectra of known IPs. This was done by cross-correlating our list of source candidates with catalogs of IPs.

For the source detection step, we used all the available BAT data taken from January 2005 to March 2007. An energy range of $15-55 \mathrm{keV}$ was chosen. The lower limit is given by the energy threshold of the detectors. The upper limit is taken to avoid the presence of strong background lines which could worsen the overall sensitivity. The data screening was performed analogously to the method presented in Ajello et al. (2008a). The final all-sky image is obtained as the weighted average of all the shorter observations. The average exposure time in our image is $3 \mathrm{Ms}$, with $1.3 \mathrm{Ms}$ minimum and $5 \mathrm{Ms}$ maximum exposure times. The final image shows Gaussian normal noise and we identified source candidates as excesses above the $3 \sigma$ level. All the candidates are then fit with the BAT point spread function (using the standard BAT tool batcelldetect) to derive the best source position. The BAT position error is between 3-7 arcmin, depending on the source intensity (see Ajello et al. 2008a). The identification of IPs from this detection list was done using the online catalog maintained by Mukai ${ }^{1}$ and allowing for each source's position error.

Out of the 29 known IPs to date, Swift/BAT detects 22 objects (see Table 1). The sources that fall below the BAT sensitivity (AE Aqr, HT Cam, DW Cnc, UU Col, DQ Her and WX Pyx) are likely either very far away, or are in a very low accretion state. All six sources except HT Cam (RXTE detection; Revnivtsev et al. 2004) were also not detected in RXTE or Integral/IBIS observations.

For each of the detected IPs, we extracted a $15-195 \mathrm{keV}$ spectrum with the method described in Ajello et al. (2008b). Here we recall the main steps. For a given source, we extract

\footnotetext{
1 http://asd.gsfc.nasa.gov/Koji.Mukai/iphome/iphome. html, version of March 2007.
} 
a spectrum from each observation for which the source is in the field of view. These spectra are corrected for residual background contamination and for vignetting. The per-pointing spectra are then (weighted) averaged to produce the final source spectrum. Thus, the final spectrum represents the average source emission over the entire survey period ( 2.5 years) although individual coverage intervals may differ.

We also extracted the light curves of all detected IPs. In most of the cases, the sources are too faint to assess any variability. The only exception is GK Per (see Fig. 2) and this source will be discussed in detail in Sect. 3.2.

\subsection{Spectral model}

The main cooling mechanism in the post-shock region is through bremsstrahlung, thus the simplest method is to fit a Bremsstrahlung spectrum to the data. The structure of the postshock region was investigated first by Aizu (1973), who provided a conversion formula to determine the white dwarf mass based on the effective temperature of the Bremsstrahlung spectrum. However, in order to estimate the mass of the accreting white dwarf more accurately, one needs a more careful modeling of the temperature and emissivity distribution in the postshock region. Woelk \& Beuermann (1996) took into account cyclotron cooling, which can be important for polars, but was shown to be unimportant for objects with surface magnetic fields less than 10 MG (like IPs). Frank et al. (2002) derived a simple analytical model under the assumption of constant pressure in the post-shock region. Suleimanov et al. (2005) extended this model to take into account how the pressure grows towards the white dwarf surface. The emergent spectrum thus is the sum of many local Bremsstrahlung spectra with their appropriate temperatures and pressures. While this model does not include reflection, Cropper et al. (1998) have shown that including the reflection changes the fit parameters and the corresponding white dwarf masses only slightly. The main difference between the model of Suleimanov et al. (2005) and that of Aizu (1973) is that Suleimanov et al. (2005) uses the cooling functions of Sutherland \& Dopita (1993), which takes thermal Bremsstrahlung, lines and $b-f$ transitions into account.

In the following we have used the model of Suleimanov et al. (2005). The free parameters are the mass of the white dwarf and a normalization constant. Since we fit spectra only above $15 \mathrm{keV}$, we do not apply any foreground absorption.

\section{Results}

\subsection{Spectral analysis}

In order to derive a WD mass estimate we fitted the model of Suleimanov et al. (2005) to the BAT IPs using XSPEC. An example is given in Fig. 1. The detection of the IP emission up to $\sim 100 \mathrm{keV}$ allows an accurate estimate of the bremsstrahlung temperature. Since the X-ray spectrum depends primarily on the WD mass, using the model of Suleimanov et al. (2005) we are able to determine directly an estimate (and its uncertainty) of the WD mass.

With the exception of MU Cam, all spectral fits show acceptable reduced $\chi^{2}$ (see Table 2 which include the errors at the $90 \%$ confidence level; the number of degrees of freedom is always 10 , based on 12 channels and 2 fit parameters). We refrained from attempting to fit more sophisticated models. Note that, in all the cases, a power law does not produce a good fit because of the clear curvature of the data.

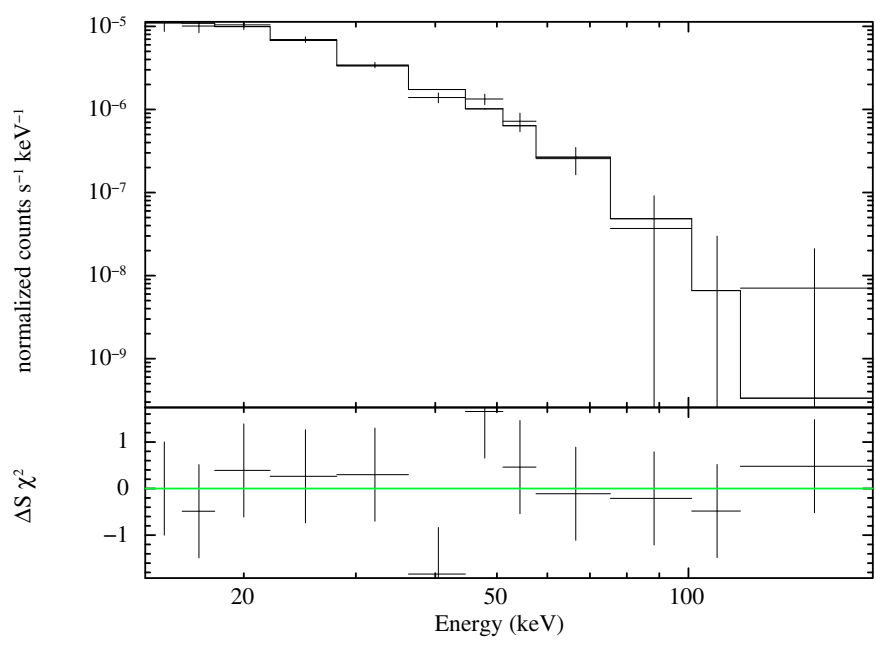

Fig. 1. Swift/BAT spectrum of GK Per, averaged over $2.5 \mathrm{yrs}$, fitted with the model described in Suleimanov et al. (2005).

\subsection{Accretion-rate dependence of GK Per}

In the simplistic case, it is assumed that the accreted mass falls freely from infinity onto the white dwarf. In reality, however, the matter does not come from infinity, but falls from the Alfvén radius $R_{\mathrm{A}}$, which in turn depends on the mass accretion rate (from Suleimanov et al. 2005):

$$
\frac{R_{\mathrm{A}}}{R_{\mathrm{WD}}} \approx 2.3\left(\frac{\dot{M}}{10^{20} \mathrm{~g} / \mathrm{s}}\right)^{-2 / 7}\left(\frac{M_{\mathrm{WD}}}{M_{\odot}}\right)^{-1 / 7}\left(\frac{R_{\mathrm{WD}}}{10^{9} \mathrm{~cm}}\right)^{5 / 7}\left(\frac{B}{10^{6} \mathrm{G}}\right)^{4 / 7}
$$

Thus, including this dependency, the white dwarf mass is:

$M_{\mathrm{WD}}\left(R_{\mathrm{A}}\right)=M_{\mathrm{WD}}(\infty)\left(1-\frac{R_{\mathrm{WD}}}{R_{\mathrm{A}}}\right)$.

Therefore, the higher the accretion rate, the smaller is $R_{\mathrm{A}}$, and thus the smaller the mass of the white dwarf which is deduced under the assumption of infinite origin.

There is yet another dependency which makes a statement on the deduced mass relative to the real mass somewhat uncertain. Accretion is only possible if $R_{\mathrm{A}}<R_{\Omega}$ (Warner 1995), where $R_{\Omega}$ is the "corotation" radius, i.e. the radius where the Keplerian angular velocity is equal to the rotation velocity of the WD (otherwise the mass would be thrown out of the system):

$$
\frac{R_{\Omega}}{R_{\mathrm{WD}}}=2.3 P_{p}^{2 / 3}(\min )\left(\frac{M_{\mathrm{WD}}}{M_{\odot}}\right)^{1 / 3}\left(\frac{R_{\mathrm{WD}}}{10^{9} \mathrm{~cm}}\right)^{-1} .
$$

Thus, fast rotating systems require higher accretion rates at the same magnetic field strength, and we measure smaller masses than they have in reality. Because of this, we think we measure only a lower limit for the mass of the following four systems (with their WD rotation period in parenthesis): GK Per (5.85 min), V709 Cas (5.12 min), DO Dra (8.83 min), V405 Aur (9.09 $\mathrm{min})$.

Figure 2 reveals that GK Per had an outburst at the beginning (January 2005) as well as at the end (Dec. 2006->Mar. 2007) of the covered observing period. GK Per is well known for its periodic outbursts, which take place every three or four years (Sabbadin \& Bianchini 1983; Šimon 2002). Brat et al. (2006) mention an increased optical brightness in Dec. 2006, about $0.6 \mathrm{mag}$ above its usual quiescent level, which is consistent with our detection of increased X-ray emission. Since GK Per is among the brightest IPs in our sample, we are able to split 
Table 2. Detected IPs with masses from XSPEC fit with the model from Suleimanov et al. (2005). The temperature is from a bremsstrahlung fit, and is provided for comparison.

\begin{tabular}{|c|c|c|c|c|c|c|c|}
\hline Name & $\begin{array}{c}k T \\
(\mathrm{keV})\end{array}$ & $\begin{array}{l}\text { Mass } \\
\left(M_{\odot}\right) \\
\end{array}$ & $\begin{array}{c}F_{14-195} \\
\left(10^{-11} \mathrm{erg} / \mathrm{cm}^{2} / \mathrm{s}\right)\end{array}$ & $\begin{array}{c}\text { Distance }^{A} \\
(\mathrm{pc})\end{array}$ & $\begin{array}{l}L_{14-195} \\
\left(10^{32} \mathrm{erg} / \mathrm{s}\right) \\
\end{array}$ & $\begin{array}{c}\dot{M} \\
\left(10^{16} \mathrm{~g} / \mathrm{s}\right)\end{array}$ & $\chi_{\mathrm{red}}^{2}$ \\
\hline FO Aqr & $15.2 \pm 1.6$ & $0.61 \pm 0.05$ & $4.7 \pm 0.5$ & $400^{1}$ & 9.00 & 29.0 & 1.4 \\
\hline XY Ari & $29.1 \pm 6.2$ & $0.96 \pm 0.12$ & $2.3 \pm 0.4$ & $270^{2}$ & 2.01 & 2.8 & 1.3 \\
\hline V405 Aur & $22.1 \pm 4.2$ & $0.89 \pm 0.13^{C}$ & $2.2 \pm 0.5$ & - & - & & 0.5 \\
\hline MU Cam & $19.6 \pm 4.7$ & $0.74 \pm 0.13$ & $1.3 \pm 0.3$ & - & - & & 1.7 \\
\hline V709 Cas & $30.0 \pm 2.5$ & $0.96 \pm 0.05^{C}$ & $7.2 \pm 0.6$ & - & - & & 1.0 \\
\hline V1025 Cen & $10.8 \pm 7.7$ & $0.46 \pm 0.31$ & $0.4 \pm 0.3$ & - & - & & 0.6 \\
\hline BG CMi & $18.9 \pm 5.5$ & $0.67 \pm 0.19$ & $2.0 \pm 0.4$ & $700^{3}$ & 11.73 & 32.3 & 0.7 \\
\hline TV Col & $21.6 \pm 2.4$ & $0.78 \pm 0.06$ & $5.0 \pm 0.5$ & $386^{4}$ & 8.92 & 18.8 & 0.9 \\
\hline TX Col & $17.0 \pm 3.2$ & $0.67 \pm 0.10$ & $1.7 \pm 0.3$ & $550^{5}$ & 6.15 & 16.9 & 0.7 \\
\hline V2306 Cyg & $20.5 \pm 5.7$ & $0.77 \pm 0.16$ & $1.2 \pm 0.3$ & - & - & & 1.0 \\
\hline DO Dra & $12.0 \pm 2.6$ & $0.50 \pm 0.11^{C}$ & $1.0 \pm 0.3$ & $155^{6}$ & 0.29 & 1.3 & 0.7 \\
\hline PQ Gem & $16.8 \pm 2.8$ & $0.65 \pm 0.09$ & $2.5 \pm 0.4$ & - & - & & 1.0 \\
\hline EX Нyа & $19.4 \pm 4.4$ & $0.66 \pm 0.17$ & $2.1 \pm 0.5$ & $64.5^{7}$ & 0.10 & 0.28 & 0.4 \\
\hline NY Lup & $39.1 \pm 4.3$ & $1.09 \pm 0.07$ & $7.1 \pm 0.6$ & $690^{8}$ & 40.45 & 25.3 & 1.1 \\
\hline V2400 Oph & $21.1 \pm 2.8$ & $0.81 \pm 0.10$ & $4.3 \pm 0.6$ & - & - & & 1.0 \\
\hline GK Per & $26.2 \pm 5.4$ & $0.90 \pm 0.12^{B, C}$ & $2.8 \pm 0.5$ & $340^{9}$ & 10.24 & 16.2 & 0.8 \\
\hline AO Psc & $13.1 \pm 1.9$ & $0.55 \pm 0.06$ & $2.8 \pm 0.4$ & $420^{10}$ & 10.13 & 38.6 & 0.7 \\
\hline V1223 Sgr & $17.0 \pm 1.3$ & $0.65 \pm 0.04$ & $8.5 \pm 0.8$ & $527^{11}$ & 28.25 & 82.0 & 1.0 \\
\hline V1062 Tau & $18.3 \pm 5.8$ & $0.72 \pm 0.17$ & $1.6 \pm 0.4$ & $500^{12}$ & 4.79 & 11.6 & 0.4 \\
\hline RX J2133 & $27.1 \pm 2.9$ & $0.91 \pm 0.06$ & $4.6 \pm 0.4$ & $>600^{13}$ & - & & 0.6 \\
\hline Swift J0713 & $21.7 \pm 4.1$ & $0.79 \pm 0.11$ & $2.5 \pm 0.4$ & - & - & & 0.9 \\
\hline IGR J00234 & $22.8_{-11.5}^{+30.0}$ & $0.85 \pm 0.39$ & $0.7 \pm 0.3$ & - & - & & 0.8 \\
\hline IGR J17303 & $37.1 \pm 4.4$ & $1.08 \pm 0.07$ & $6.6 \pm 0.6$ & - & - & & 1.1 \\
\hline
\end{tabular}

${ }^{A}$ References to the distances: 1. McHardy et al. (1987); 2. Littlefair et al. (2001); 3. Berriman (1987); 4. McArthur et al. (2001); 5. Buckley \& Tuohy (1989); 6. Mateo et al. (1991); 7. Beuermann et al. (2003); 8. de Martino et al. (2006); 9. Warner (1987); 10. Hellier et al. (1991); 11. Beuermann et al. (2004); 12. Szkody \& Silber (1996); 13 Bonnet-Bidaud et al. (2006).

${ }^{B}$ All numbers correspond to the measured spectrum during the low-flux period; but see Sect. 3.2.

${ }^{C}$ The derived mass is likely a lower limit due to the fast rotation of the WD; see Sect. 3.1.
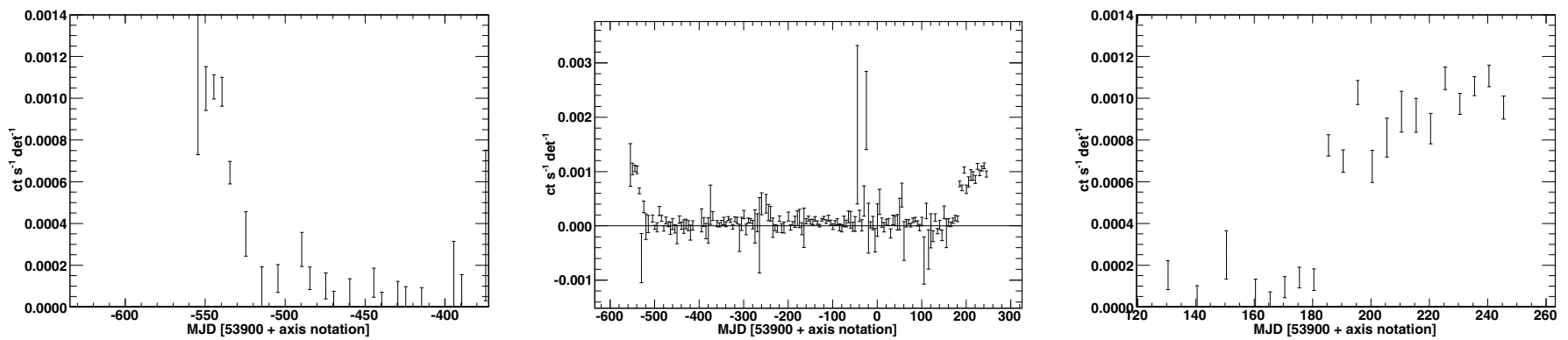

Fig. 2. Light curves of GK Per in the $15-195 \mathrm{keV}$ band with a binning of 5 days. The middle panel shows the full observation while the left panel shows the outburst at the beginning and the right panel that at the end of the covered observing period.

the data into three different periods, namely the low-flux period as well as the two outbursts, and derive the mass separately for these periods.

The results in Table 3 show that we measure lower masses at higher flux. While our data are not sufficient to demonstrate that the mass estimates quantitatively follow that expected on the basis of the expected change in $R_{\mathrm{A}}$, the change is suggestive of being due the dependence on the accretion rate according to Eqs. (1) and (2). Note the systematically higher mass estimate if we were not been able to distinguish the different flux levels. Only the mass derived during the low-flux (low-accretion) period of GK Per should be considered - the last row in Table 3 is for illustrative purposes only. The values as derived during the outbursts are similar to those of Suleimanov et al. (2005), suggesting that those data were also taken during periods of
Table 3. Comparison of mass estimates for different sections of the light curve of GK Per.

\begin{tabular}{lcc}
\hline \hline Part of light curve & Mass $\left(M_{\odot}\right)$ & $\chi_{\text {red }}^{2}$ \\
\hline Low-flux period, without outbursts & $0.90 \pm 0.12$ & 0.78 \\
Jan. 2005 outburst & $0.74 \pm 0.05$ & 1.66 \\
Mar. 2007 outburst & $0.67 \pm 0.03$ & 1.24 \\
Sum of the total period & $0.78 \pm 0.05$ & 0.79 \\
\hline
\end{tabular}

increased accretion. We finally note that for all other sources of Table 2 the mass estimates are unlikely to be affected by high accretion rates, i.e. the source being in outburst.

Assuming that the magnetic field (and the mass) does not change significantly, we attempted to determine the accretion 

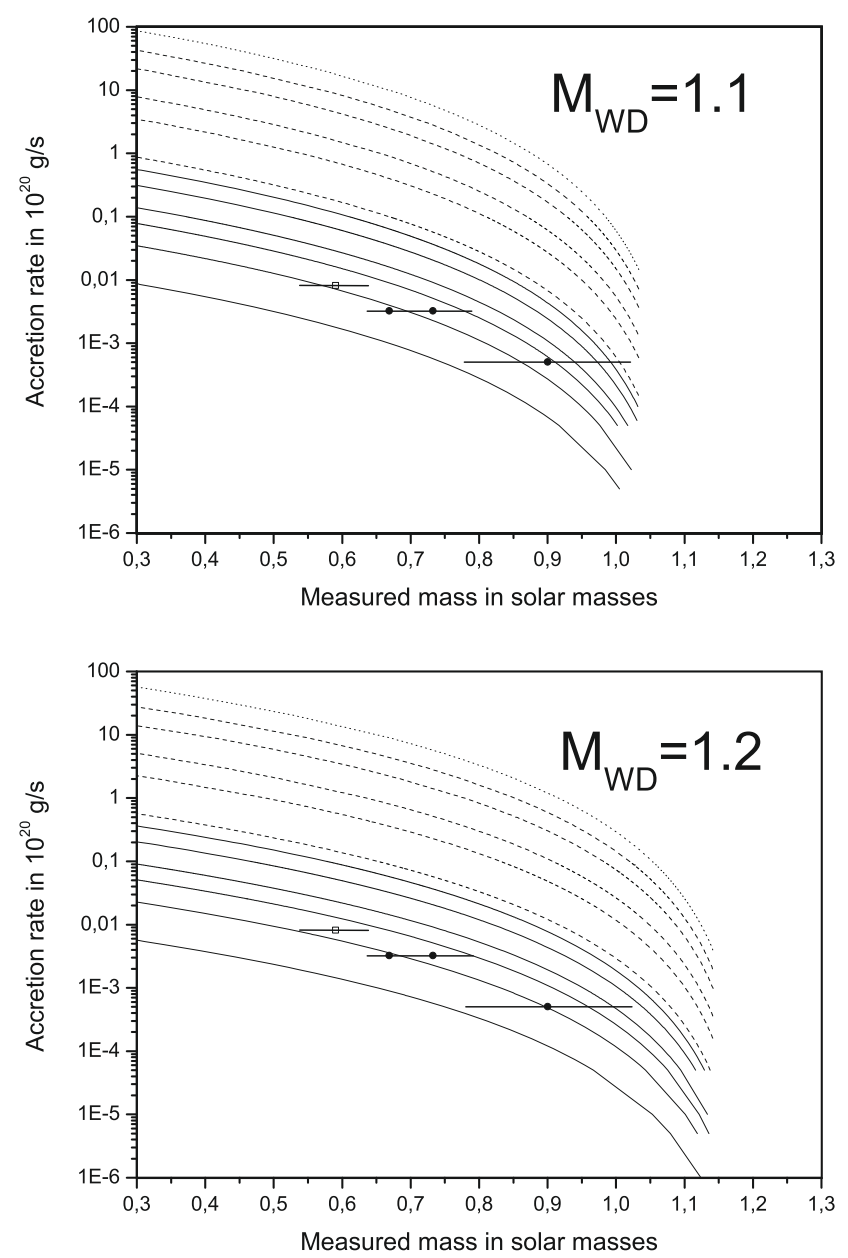

Fig. 3. Relation between measured mass and accretion rate for different magnetic fields for $M_{\mathrm{WD}}=1.1 M_{\odot}$ and $M_{\mathrm{WD}}=1.2 M_{\odot}$. The values of this work are shown (filled circles) with accretion rates from Warner (1995). The parallel curves are for different values of the magnetic field, namely $0.1,0.2,0.3,0.4,0.6,0.8,1,2,3,5,7$ and $10 \mathrm{MG}$, respectively (from bottom to top). The open square represents the measured mass and its corresponding accretion rate derived by Suleimanov et al. (2005). The accretion rate has its first limit from the constraint that the system must have an accretion rate lower than $0.01 \times 10^{20} \mathrm{~g} / \mathrm{s}$ at quiescence, because of its life time.

rate via Eqs. (1)-(3), using the rotation period of $351 \mathrm{~s}$ Watson et al. (1985). In Fig. 3 the three mass estimates from Table 3 are shown together with the generally adopted values for the mass accretion rate of GK Per in its different states (Warner 1995), namely $4 \times 10^{16} \mathrm{~g} / \mathrm{s}$ at quiescence and $23 \times 10^{16} \mathrm{~g} / \mathrm{s}$ in outburst. We also add the measurement of $M=0.6 M_{\odot}$ from Suleimanov et al. (2005). In order to be consistent, all four measurements should fall onto one particular line for the magnetic field of the white dwarf. Testing white dwarf masses between 0.6-1.3 $M_{\odot}$, we find that the mass of the white dwarf in GK Per must be definitely higher than $0.9 M_{\odot}$. A mass of $1.15 M_{\odot}$ provides the best match, and we present the graphs for 1.1 and $1.2 M_{\odot}$ to show how sensitive the mass estimate is (note, however, that this hinges on the assumption of the accretion rate). For $1.1 M_{\odot}$ the data points are flatter than the corresponding curve of $B=$ $0.2 \mathrm{MG}$, while for $1.2 M_{\odot}$ the data points are already steeper than the nearest $B=$ const. curve. The online material provides the remaining figures with white dwarf masses of $0.9 M_{\odot}, 1.0 M_{\odot}$ and $1.3 M_{\odot}$. Our accretion rate corrected mass of the white dwarf of GK Per of $1.15 M_{\odot}$ compares well with the lower limit of
$0.9 M_{\odot}$ derived by Morales-Rueda et al. (2002). However, the implied magnetic field of $0.2 \mathrm{MG}$ is at the low end of what is believed to be the canonical magnetic field strength of IPs, namely 1-10 MG (de Martino et al. 2004).

The canonical way of deriving the accretion rate is via the X-ray luminosity, i.e. using the relation $L_{X}=0.1 \times \epsilon \times$ $G \times M_{\mathrm{WD}} \times \dot{M} / R_{\mathrm{WD}}$, where $\epsilon$ is the bolometric correction to the X-ray luminosity in the $14-195 \mathrm{keV}$ band, the factor 0.1 accounts for the ratio of X-ray to total accretion luminosity, and we assume the conversion efficiency of gravitational binding energy to radiation being equal to 1 for WD surface accretion, as well as isotropic emission. For a bremsstrahlung spectrum of 20 (40) $\mathrm{keV}, \epsilon=0.33$ (0.53). Using our mass estimate of $0.90(1.15) M_{\odot}$ and the corresponding radius of $6200(3500) \mathrm{km}$ (according to the relativistic Fermi gas), we obtain $\dot{M}=1.7(0.7) \times 10^{16} \mathrm{~g} / \mathrm{s}$. This is somewhat lower than according to the typical values used above, and, if true, would suggest an even lower magnetic field. It seems obvious that the combination of the accretion rate estimate from $L_{\mathrm{X}}$ with the dependencies according to Eqs. (1)-(3) is not fully consistent with our general belief of $B \sim 1-10 \mathrm{MG}$ in IPs.

\subsection{Comparison with other methods of mass determination}

The canonical method of determining the WD mass in CVs is by measuring the radial velocities of optical emission lines. However, this method suffers from two main problems. First, the uncertainty in the inclination of the system leads to a wide range in mass. Second, in magnetic systems the velocities along the collimating field lines dominate the radial velocities.

An alternative method is to measure the mass ratio and infer the mass of the secondary by modeling the infrared light curves (e.g. Allan et al. 1996). Again, the uncertainty in the inclination limits the accuracy of the mass determination.

Eclipsing systems offer a unique opportunity to measure the mass more accurately due to the relatively small uncertainty in the inclination of the binary system. Moreover, in eclipsing systems the radius of the white dwarf can be determined, thus providing yet another method (though rarely applicable due to the few eclipsing systems) to determine the WD mass.

\subsubsection{The whole sample}

In Table 4 and Fig. 4 we compare the mass estimates obtained by our analysis to previous mass estimates via radial velocity studies. In general, there is good agreement, with no systematic effect visible. A detailed comparison with respect to individual sources is given below.

While we compare our results to those obtained with IBIS onboard INTEGRAL (see below), we choose not to discuss the differences (Table 4) of our results to those obtained by Ginga, ASCA and RXTE due to the systematic, and unexplained, differences for XY Ari (Ramsay et al. 1998). In the next section we discuss each object.

\subsubsection{Discussion of individual objects}

The mass of $0.96 \pm 0.12 M_{\odot}$ for XY Ari matches very well the value of $1.04 \pm 0.13 M_{\odot}$ from Hellier (1997). This is particularly encouraging given the systematic differences in previous estimates as discussed in Ramsay et al. (1998) - see the Introduction. Since XY Ari is the only eclipsing IP, its optically determined mass is generally assumed to be secure. The fact that 
Table 4. Comparison of our mass estimates with those from previous X-ray observations

\begin{tabular}{llllllll}
\hline \hline Name & BAT $^{1}$ & $\begin{array}{l}\text { RXTE }^{2} \\
M_{\odot}\end{array}$ & $\begin{array}{l}\mathbf{R X T E}_{\odot} \\
M_{\odot}\end{array}$ & $\begin{array}{l}\text { GINGA }^{4} \\
M_{\odot}\end{array}$ & $\begin{array}{l}\text { ASCA }^{5} \\
M_{\odot}\end{array}$ & $\begin{array}{l}\text { Other methods }^{6} \\
M_{\odot}\end{array}$ & References $^{7}$ \\
\hline FO Aqr & $0.61 \pm 0.05$ & $0.60 \pm 0.05$ & 0.88 & 0.92 & 1.05 & & \\
XY Ari & $0.96 \pm 0.12$ & & 0.97 & & & $1.04 \pm 0.13$ & {$[1]$} \\
V405 Aur & $0.89 \pm 0.13$ & $0.90 \pm 0.10$ & 1.10 & & $>0.54$ & & \\
V709 Cas & $0.96 \pm 0.05$ & $0.90 \pm 0.10$ & 1.08 & & & & \\
V1025 Cen & $0.46 \pm 0.31$ & & 0.60 & & & $0.8 \pm 0.2$ & {$[2]$} \\
BG CMi & $0.67 \pm 0.19$ & $0.85 \pm 0.12$ & 1.20 & 1.09 & & & \\
TV Col & $0.78 \pm 0.06$ & $0.84 \pm 0.06$ & 0.96 & 1.30 & 0.51 & $0.75 \pm 0.15$ & {$[3]$} \\
TX Col & $0.67 \pm 0.10$ & $0.70 \pm 0.30$ & 0.73 & 0.48 & 0.66 & & \\
DO Dra & $0.50 \pm 0.11$ & $0.75 \pm 0.05$ & & & & $0.83 \pm 0.10$ & {$[4]$} \\
PQ Gem & $0.65 \pm 0.09$ & $0.65 \pm 0.20$ & & 1.29 & & & \\
EX Hya & $0.66 \pm 0.13$ & $0.50 \pm 0.05$ & 0.45 & 0.46 & 0.48 & $0.49 \pm 0.13$ & {$[5]$} \\
NY Lup & $1.09 \pm 0.07$ & & & & & $>0.5$ & {$[6]$} \\
V2400 Oph & $0.81 \pm 0.10$ & $0.59 \pm 0.05$ & 0.71 & & 0.68 & & \\
GK Per & $0.90 \pm 0.12$ & $0.59 \pm 0.05$ & & & 0.52 & $0.9 \pm 0.2,>0.87$ & {$[7],[8]$} \\
AO Psc & $0.55 \pm 0.06$ & $0.65 \pm 0.05$ & 0.60 & 0.56 & 0.40 & & \\
V1223 Sgr & $0.65 \pm 0.04$ & $0.95 \pm 0.05$ & 1.10 & & 1.28 & $0.4-0.6$ & {$[2]$} \\
V1062 Tau & $0.72 \pm 0.17$ & $1.00 \pm 0.20$ & 0.90 & & & & \\
\hline
\end{tabular}

References: 1: this work, BAT; 2: Suleimanov et al. (2005); 3: Ramsay (2000); 4: Cropper et al. (1999); 5: Ezucka, Ishida (1999); 6: Other methods of determination of mass and the last column are the references of them. 7: References: [1] Hellier (1997); [2] Penning (1985); [3] Hellier (1993); [4] Haswell et al. (1997); [5] Hoogerwerf et al. (2004); [6] de Martino et al. (2006); [7] Crampton et al. (1986); [8] Morales-Rueda et al. (2002).

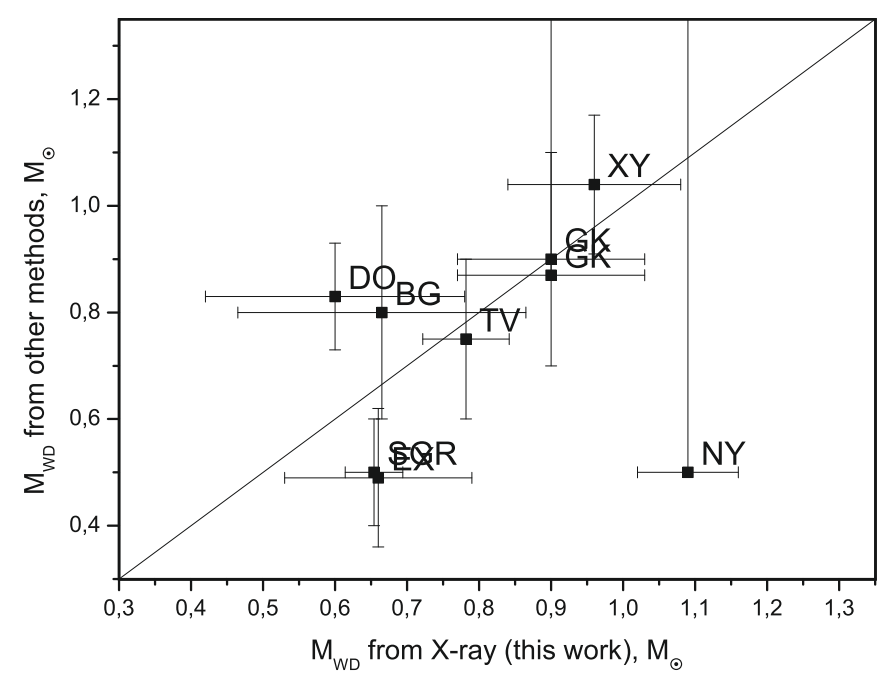

Fig. 4. Comparison of the measured white dwarf masses with the ones from other methods (values from Table 4). The source names are abbreviated; the labels of V1223 Sgr and EX Hya overlap.

our mass estimate matches so well suggests that our analysis does not suffer from major systematic uncertainties.

Penning (1985) derives a mass range of $0.6-1.0 M_{\odot}$ for the WD in BG CMi. Our value of $0.72 \pm 0.14 M_{\odot}$ falls in that range.

For the system TV Col, Hellier (1993) determined a mass of $0.75 \pm 0.15 M_{\odot}$, which agrees very well with our estimate of $0.78 \pm 0.06 M_{\odot}$.

In DO Dra our WD mass estimate is $0.50 \pm 0.11 M_{\odot}$. Haswell et al. (1997) find a mass of $0.83 \pm 0.10 M_{\odot}$, which is substantially different to our value. Mateo et al. (1991) also find a mass of $0.83 \pm 0.10 M_{\odot}$. The difference could be the result of the short spin period of the WD (see Sect. 3.2), thus making our estimate a lower limit. However, our mass estimates of the other WDs with short spin period are not as far below the optical estimates.

Using data of the first months of BAT observations, a significantly higher mass $\left(0.83 \pm 0.17 M_{\odot}\right)$ is deduced for EX Hya than for the rest of the time - although the count rate does not differ in these periods. If the first 100 days are ignored, a mass of $0.66 \pm$ $0.13 M_{\odot}$ is derived. While we cannot completely exclude a statistical fluctuation which leads to a change in the spectrum, one possible physical reason for the strange behavior could be that this system has a much lower mass accretion rate than the others. In the model of Suleimanov et al. (2005) cyclotron cooling plays a role for a $<0.01 \mathrm{~g} / \mathrm{s} / \mathrm{cm}^{2}$, so if the accretion rate were lower, this could provide an explanation. Hoogerwerf et al. (2004) measured a mass for the WD in EX Hya of $0.49 \pm 0.13 M_{\odot}$, which is comparable within the errors with our value.

In the system NY Lup we obtain a mass of $1.12 \pm 0.06 M_{\odot}$. This value is consistent with the lower limit of $0.5 M_{\odot}$, which Martino et al. (2006) determined.

For V1223 Sgr a WD mass of $0.65 \pm 0.04 M_{\odot}$ was derived. Penning (1985) gives a range of 0.4-0.6 $M_{\odot}$, which fits our value.

The accretion-corrected mass of $1.15 \pm 0.15 M_{\odot}$ for GK Per is within the error bars of the values from other methods: $0.9 \pm$ $0.2 M_{\odot}$ from Crampton et al. (1986) and the lower limit $M>$ $0.87 \pm 0.24 M_{\odot}$ from Morales-Rueda et al. (2002).

\subsubsection{Comparison with INTEGRAL/IBIS data}

Barlow et al. (2006) and very recently Landi et al. (2009) provided a table with temperatures derived from Integral/IBIS data. The method of Aizu (1973) provides a direct conversion from the bremsstrahlung temperature to mass of the white dwarf. which we use to derive the masses as listed in Table 5. The comparison of these masses to those derived here using Swift/BAT shows, in general, very good agreement (Table 5. The only outlier is MU Cam where our temperature (and mass) is substantially greater. However, in a recent analysis of XMM-Newton and INTEGRAL data, Staude et al. (2008) conclude that the estimate of Barlow et al. (2006) seems too low. These authors attempted a combined XMM-INTEGRAL fit, and arrive at a temperature of $35 \pm 10 \mathrm{keV}$, which they describe as "convincing agreement with the INTEGRAL data points". Though the Swift/BAT spectrum for MU Cam is the worst in terms of reduced $\chi^{2}$, we are 
Table 5. Comparison of masses and effective temperatures with a remsstrahlung model from BAT (this work, model: Suleimanov et al. 2005) and INTEGRAL/IBIS by (Barlow et al. 2006) and (Landi et al. 2009). 1: Masses, derived from the temperatures with the model of Aizu (1973). 2: Bremsstrahlung temperature.

\begin{tabular}{lllllll}
\hline \hline Name & \multicolumn{2}{c}{ BAT/this work } & \multicolumn{2}{c}{ IBIS/Barlow } & \multicolumn{2}{c}{ IBIS/Landi } \\
& mass $\left(M_{\odot}\right)$ & $k T(\mathrm{keV})$ & mass $\left(M_{\odot}\right)^{1}$ & $k T(\mathrm{keV})^{2}$ & $\operatorname{mass}_{\left(M_{\odot}\right)^{1}} k T(\mathrm{keV})^{2}$ \\
\hline FO Aqr & $0.61 \pm 0.05$ & $15.2_{-1.5}^{+1.7}$ & - & - & $1.06_{-0.51}^{+0.38}$ & $29.7_{-16.6}^{+70.1}$ \\
MU Cam & $0.74 \pm 0.13$ & $19.6 \pm 4.7$ & $0.37 \pm 0.17$ & $8.1 \pm 4.7$ & - & - \\
V709 Cas & $0.96 \pm 0.05$ & $30.0 \pm 2.5$ & $0.88 \pm 0.06$ & $23.3 \pm 2.2$ & $0.95_{-0.08}^{+0.07}$ & $25.6_{-2.4}^{+2.7}$ \\
NY Lup & $1.12 \pm 0.06$ & $39.1 \pm 4.3$ & $0.99 \pm 0.06$ & $27.1 \pm 2.2$ & - & - \\
V2400 Oph & $0.81 \pm 0.10$ & $21.1 \pm 2.8$ & $0.73 \pm 0.05$ & $18.6 \pm 1.4$ & - & - \\
GK Per & $0.90 \pm 0.12$ & $26.2 \pm 5.4$ & $1.00 \pm 0.40$ & $28.7 \pm 15.6$ & $0.87_{-0.21}^{+0.25}$ & $23.0_{-6.5}^{+9.2}$ \\
V1223 Sgr & $0.65 \pm 0.04$ & $17.0 \pm 1.3$ & $0.74 \pm 0.07$ & $18.8 \pm 1.2$ & - & - \\
RX J2133 & $0.91 \pm 0.06$ & $27.1 \pm 2.9$ & $0.90 \pm 0.12$ & $23.8 \pm 4.3$ & - & - \\
IGR J00234+6141 & $0.85 \pm 0.39$ & $22.8_{-11.5}^{+30.5}$ & $0.64 \pm 0.18$ & $15.9 \pm 5.1$ & - & - \\
IGR J17303-0601 & $1.08 \pm 0.07$ & $37.1 \pm 4.4$ & $0.98 \pm 0.14$ & $26.7 \pm 4.8$ & $1.10_{-0.16}^{+0.21}$ & $31.6_{-7.8}^{+12.7}$ \\
\hline
\end{tabular}

confident that our measurement does not suffer from large systematic uncertainties.

\section{Conclusions}

An accurate determination of white dwarf masses in IPs is important for our understanding of the accretion geometry and binary evolution. However, this has so far been complex because of the difficulty in measuring the radial velocities and inclination of the systems. The main advantage of using X-ray measurements is that they allow a direct measurement of the WD mass without relying on the above parameters.

Indeed, since bremsstrahlung is the main cooling mechanism in the post-shock region, it is possible to relate the temperature of the IP's X-ray spectrum to the WD mass (e.g. Aizu 1973; Suleimanov et al. 2005). Using this method, we determined WD masses for a complete sample of 22 IPs detected in the ongoing Swift/BAT survey. For 6 objects, this represents the only available mass estimate.

All mass estimates are in good agreement with previously estimated WD masses and in general, thanks to the good signalto-noise ratio, are more accurate. In particular, we reach surprisingly good agreement for the only eclipsing IP, XY Ari, suggesting that our analysis does not suffer from major systematic errors.

Detailed analysis of the light curve of GK Per shows that BAT detects the dependence of the source flux on the accretion rate. While this has been expected for a long time, to our knowledge this effect has never yet been supported by observational evidence.

Acknowledgements. We are grateful to Valery Suleimanov for providing his model and for his help. J.B. acknowledges extensive discussions with H. Ritter and M. Revnivtsev. M.A. acknowledges funding from the DFG Leibniz-Prize to Prof. G. Hasinger (HA 1850/28-1). We thank the referee for the quick response. This research has made use of the NASA/IPAC extragalactic Database (NED) which is operated by the Jet Propulsion Laboratory, of data obtained from the High Energy Astrophysics Science Archive Research Center (HEASARC) provided by NASA's Goddard Space Flight Center and of the SIMBAD Astronomical Database.

\section{References}

Aizu, K. 1973, Prog. Theor. Phys., 49, 1184

Ajello, M., Greiner, J., Kanbach, G., et al. 2008a, ApJ, 678, 102

Ajello, M., Rau, A., Greiner, J., et al. 2008b, ApJ, 673, 96

Allan, A., Hellier, C., \& Ramseyer, T. F. 1996, MNRAS, 282, 699

Barlow, E. J., Knigge, C., Bird, A. J., et al. 2006, MNRAS, 372, 224
Barthelmy, S. D. 2004, in X-Ray and Gamma-Ray Instrumentation for Astronomy XIII, ed. K. A. Flanagan, \& O. H. W. Siegmund, Proc. SPIE, 5165,175

Berriman, G. 1987, A\&AS, 68, 41

Beuermann, K., Harrison, T. E., McArthur, B. E., Benedict, G. F., \& Gänsicke, B. T. 2003, A\&A, 412, 821

Beuermann, K., Harrison, T. E., McArthur, B. E., Benedict, G. F., \& Gänsicke, B. T. 2004, A\&A, 419, 291

Bonnet-Bidaud, J. M., Mouchet, M., de Martino, D., Silvotti, R., \& Motch, C. 2006, A\&A, 445, 1037

Brat, L., Hudec, R., Simon, V., et al. 2006, The Astronomer's Telegram, 965, 1 Buckley, D. A. H., \& Tuohy, I. R. 1989, ApJ, 344, 376

Crampton, D., Fisher, W. A., \& Cowley, A. P. 1986, ApJ, 300, 788

Cropper, M., Ramsay, G., \& Wu, K. 1998, MNRAS, 293, 222

Cropper, M., Wu, K., Ramsay, G., \& Kocabiyik, A. 1999, MNRAS, 306, 684

de Martino, D., Matt, G., Belloni, T., et al. 2004, Nucl. Phys. B Proc. Suppl., 132,693

de Martino, D., Bonnet-Bidaud, J.-M., Mouchet, M., et al. 2006, A\&A, 449, 1151

Frank, J., King, A., \& Raine, D. J. 2002, Accretion Power in Astrophysics: Third Edition, ed. J. Frank, A. King, \& D. J. Raine (Cambridge, UK: Cambridge University Press), 398

Haswell, C. A., Patterson, J., Thorstensen, J. R., Hellier, C., \& Skillman, D. R. 1997, ApJ, 476, 847

Hellier, C. 1993, MNRAS, 264, 132

Hellier, C. 1997, MNRAS, 291, 71

Hellier, C. 2002, in The Physics of Cataclysmic Variables and Related Objects, ed. B. T. Gänsicke, K. Beuermann, \& K. Reinsch, ASP Conf. Ser., 261, 92

Hellier, C., Cropper, M., \& Mason, K. O. 1991, MNRAS, 248, 233

Hoogerwerf, R., Brickhouse, N. S., \& Mauche, C. W. 2004, ApJ, 610, 411

Ishida, M. 1991, Ph.D. Thesis, Univ. Tokyo

King, A., \& Lasota, J. 1979, MNRAS, 188, 653

Landi, R., Bassani, L., Dean, A. J., et al. 2009, MNRAS, 392, 630

Littlefair, S. P., Dhillon, V. S., \& Marsh, T. R. 2001, MNRAS, 327, 669

Markwardt, C. B., Tueller, J., Skinner, G. K., et al. 2005, ApJ, 633, L77

Mateo, M., Szkody, P., \& Garnavich, P. 1991, ApJ, 370, 370

McArthur, B. E., Benedict, G. F., Lee, J., et al. 2001, ApJ, 560, 907

McHardy, I. M., Pye, J. P., Fairall, A. P., \& Menzies, J. W. 1987, MNRAS, 225, 355

Morales-Rueda, L., Still, M. D., Roche, P., Wood, J. H., \& Lockley, J. J. 2002, MNRAS, 329, 597

Penning, W. R. 1985, ApJ, 289, 300

Ramsay, G. 2000, MNRAS, 314, 403

Ramsay, G., Cropper, M., Hellier, C., \& Wu, K. 1998, MNRAS, 297, 1269

Revnivtsev, M., Sazonov, S., Jahoda, K., \& Gilfanov, M. 2004, A\&A, 418, 927

Rothschild, R. E., Gruber, D. E., Knight, F. K., et al. 1981, ApJ, 250, 723

Sabbadin, F., \& Bianchini, A. 1983, A\&AS, 54, 393

Staude, A. Schwope, A. D., Schwarz, R., et al. 2008, A\&A, 486, 899

Suleimanov, V., Revnivtsev, M., \& Ritter, H. 2005, A\&A, 435, 191

Sutherland, R. S., \& Dopita, M. A. 1993, ApJS, 88, 253

Szkody, P., \& Silber, A. 1996, AJ, 112, 289

Šimon, V. 2002, A\&A, 382, 910

Warner, B. 1987, MNRAS, 227, 23

Warner, B. 1995, Cataclysmic variable stars, Cambridge Astrophysics Series (Cambridge, New York: Cambridge University Press)

Watson, M. G., King, A. R., \& Osborne, J. 1985, MNRAS, 212, 917

Woelk, U., \& Beuermann, K. 1996, A\&A, 306, 232 